\title{
Practical Use of Acoustic Rhinometry for Quantitative Assessment of Nasal Obstruction in an Allergic Model Using Macaque Monkeys
}

\author{
Fusako Mitsunaga ${ }^{1,2^{*}}$, Shin Nakamura ${ }^{2,3}$, Motofumi Ohki ${ }^{4}$ \\ ${ }^{1}$ Kaizu Laboratory, Biomedical Institute, NPO Primate Agora, Kaizu, Japan \\ ${ }^{2}$ Intelligence and Technology Lab Inc., Kaizu, Japan \\ ${ }^{3}$ Biomedical Institute, NPO Primate Agora, Inuyama, Japan \\ ${ }^{4}$ Department of Otorhinolaryngology, Kitasato University Medical Center, Kitamoto, Japan \\ Email: *mitsunaga@primate-agora.jp
}

How to cite this paper: Mitsunaga, F., Nakamura, S. and Ohki, M. (2017) Practical Use of Acoustic Rhinometry for Quantitative Assessment of Nasal Obstruction in an Allergic Model Using Macaque Monkeys. Journal of Biosciences and Medicines, 5 , 27-32.

https://doi.org/10.4236/jbm.2017.511004

Received: October 16, 2017

Accepted: November 17, 2017

Published: November 20, 2017

Copyright (๑) 2017 by authors and Scientific Research Publishing Inc. This work is licensed under the Creative Commons Attribution International License (CC BY 4.0).

http://creativecommons.org/licenses/by/4.0/

(c) (i) Open Access

\begin{abstract}
Nasal obstruction, a primary symptom of allergic rhinitis, results in breathing difficulties and decreases quality of life in patients. Quantitative methods are required for diagnosis of nasal obstruction to accurately assess drug efficacy, not only in patients but also in animal models. Acoustic rhinometry is a non-invasive technique to measure nasal airway patency using acoustic reflections. To date, little information on its application in non-human primates (NHP) has been accumulated. Macaque monkeys are a practical NHP model because of their high similarity with humans in their biomedical nature, including genomic and immunologic events and morphology. We established an allergic rhinitis model of cynomolgusmacaque (Macaca fascicularis) sensitized with Japanese cedar pollen and applied acoustic rhinometry to measure nasal congestion. The extent of nasal congestion followed by allergen challenge was successfully quantified as the minimal cross-sectional area (MCA) of the nasal passage for the first time in an NHP allergic model. We also applied this acoustic technique to evaluate the efficacy of an antihistamine drug, fexofenadine. The acoustic assessment achieved quantitative evaluation of the efficacy of fexofenadine on nasal congestion in a macaque allergic model.
\end{abstract}

\section{Keywords}

Non-Human Primate, Pollinosis, Nasal Congestion, Allergic Rhinitis, Antihistamine

\section{Introduction}

Nasal obstruction is a primary symptom of allergic rhinitis. It results in breathing 
difficulties and causes sleep disorders and/or mouth breathing [1]. The degree of nasal congestion is usually assessed by patient complaints [2], which differ markedly among patients. Rhinoscopy is useful for the direct observation of nasal congestion, and rhinoscopic criteria are defined appropriately in the Practical Guideline for the Management of Allergic Rhinitis in Japan [2] [3]. However, rhinoscopy is not quantitative, although it is effective in identifying and differentiating nasal events. Additional advanced methods are required for quantitative diagnosis of nasal symptoms to accurately assess drug efficacy, not only in patients but also in animal models. Acoustic rhinometry is a non-invasive technique to measure nasal airway patency using acoustic reflections [4] [5]. This technique measures the geometry of a cavity as a plot of the cross-sectional area against distance, independent of airflow. Acoustic rhinometry would enable accurate diagnosis by providing a reproducible and quantitative assessment of nasal congestion.

Acoustic rhinometry has been used in experimental guinea pigs [6], dogs, cats [7], and rats [8]. However, little information on its application in non-human primates (NHPs) has been accumulated to date. Macaque monkeys are a practical NHP model because of their high similarity with humans in their biomedical nature, including genomic and immunologic events [9]. Moreover, compared with rodents or carnivores, macaques closely resemble humans in the morphology of their nasal cavities [10]. Establishing an acoustic rhinometry technique for macaques provides an invaluable contribution to non-clinical studies on the efficacy of drugs for allergies and related diseases.

In the present study, we established an acoustic rhinometry technique for the quantitative assessment of nasal obstruction in an allergic model of cynomolgus macaques (Macaca fascicularis) sensitized with Japanese cedar pollen. We also applied this acoustic technique to evaluate the efficacy of an antihistamine drug, fexofenadine, using the allergic model of cynomolgus macaques.

\section{Animals and Methods}

Cynomolgus macaques were sensitized to the pollen allergen Japanese cedar. A crude extract of Japanese cedar pollen (Biostir Inc., Osaka, Japan) and Imject Alum Adjuvant (Thermo Fisher Scientific, Waltham, MA, USA) were mixed to make a suspension of $0.5 \mu \mathrm{g} / \mathrm{mL}$ Cry j 1 and $20 \mathrm{mg} / \mathrm{mL}$ aluminum hydroxide. The suspension was subcutaneously injected $(1 \mathrm{~mL} / \mathrm{head})$ into the backs of monkeys more than 5 times at intervals of 1 month.

The plasma levels of allergen-specific IgE were monitored by a sensitive ELISA utilizing a biotinyltyramide amplification system (PerkinElmer Inc., Waltham, MA, USA). Two male monkeys (14 - 18 years old, $6.2-8.8 \mathrm{~kg} \mathrm{BW}$ ) with raised allergen-specific IgE titers more than 2 -fold greater than basal levels and that had developed allergic rhinitis were used in this study.

A crossover experiment to evaluate the antihistamine drug fexofenadine was performed on nasal congestion utilizing acoustic rhinometry. One hour after oral administration of fexofenadine hydrochloride $(30 \mathrm{mg} / \mathrm{kg}$ BW, Tokyo 
Chemical Industry Co., Ltd., Tokyo, Japan) suspended in $0.5 \%$ methyl cellulose solution (Wako Pure Chemical Industries, Ltd., Osaka, Japan) or a vehicle (0.5\% methyl cellulose solution), the nasal cavities were challenged with the allergen using a nasal sprayer. Prior to each challenge, nasal cavities were pre-treated with $1.5 \% \mathrm{~N}$-Acetyl-L-cysteine to perturbate mucus layer and washed with saline. At the challenge, a crude extract of Japanese cedar pollen (including 2.5 $\mu \mathrm{g} / \mathrm{mL}$ of Cry j1) was sprayed into the nasal cavities at $0.2 \mathrm{ml} / \mathrm{site}$.

A cross-sectional area of the nasal cavity was measured with an acoustic rhinometer (A1, GM Instruments Ltd., Kilwinning, UK) and nasal congestion was observed with a rhinoscope (TESARA AE-C1 Camera System, AVS Co. Ltd., Tokyo, Japan) just before (pre-challenge) and 10 to 20 minutes after challenge (post-challenge). For rhinometry, the nosepieces of the acoustic rhinometer were made of $1000-\mu \mathrm{L}$ pipette tips and silicone resin to adjust to the size of the monkeys' nostrils. At measurement, the nosepiece was carefully attached to the nostril to prevent leaks between the nosepiece and tissue and deformation of the nasal cavity.

Monkeys were anesthetized at subcutaneous sensitization, oral administration, and nasal challenge tests with ketamine hydrochloride (3 or $10 \mathrm{mg} / \mathrm{kg} \mathrm{BW}$, depending on the duration of immobilization). All experimental procedures were approved by the institutional animal ethics committee in accordance with the institutional guide based on the Guide for the Care and Use of Laboratory Animals by the National Research Council (US) Institute for Laboratory Animal Research. Data were expressed as mean \pm standard deviation (SD). Comparisons were made using a Student's t-test if the variance was equal and Welch's t-test if the variance was unequal.

\section{Results and Discussion}

To our knowledge, the extent of nasal congestion was successfully quantified as the minimal cross-sectional area (MCA) of the nasal passage for the first time in an NHP allergic model. The allergic reaction in a type I allergy occurs within minutes. Peak nasal congestion in the early phase was usually observed 10 to 20 minutes after allergen challenge. Thus, we compared the pre- and post-challenge MCAs. In case of vehicle administration, the post-challenge MCA decreased by more than $50 \%$ of the pre-challenge value (Figure $1(\mathrm{a})$ ). Coinciding with the acoustic measurement, the post-challenge rhinoscopic images indicated nasal congestion (Figure 1(c)). The notch of the MCA was at the distal end of the ethmoturbinate in most cases [10].

When fexofenadine was administrated one hour prior to the allergen challenge, the post-challenge MCA did not decrease as much as that in the case of vehicle administration compared to the pre-challenge MCA (Figure 1(b)). Rhinoscopic images also demonstrated that nasal congestion was ameliorated by fexofenadine administration. The tip of the ethmoturbinate was visible in the middle of the rhinoscope view field in fexofenadine administration (Figure 1(d)), whereas the ethmoturbinate was hardly recognized on vehicle administration 




(a)

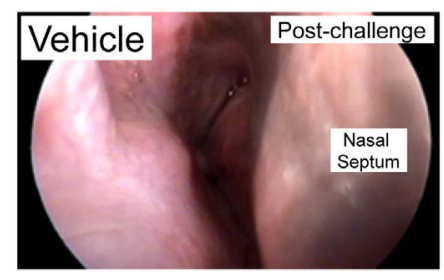

(c)

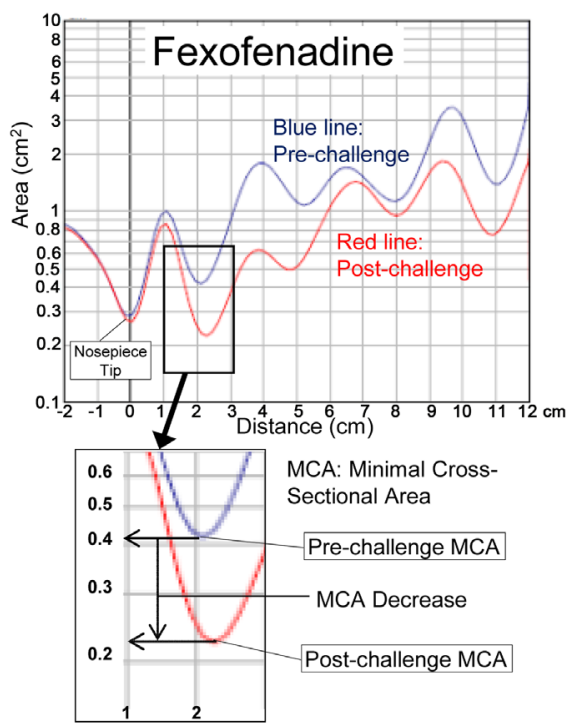

(b)



(d)

Figure 1. Typical acoustic rhinometric measurements ((a), (b)) and corresponding rhinoscopic images of the right nasal cavity ((c), (d)) of a pollinosis model of a cynomolgus macaque during an allergen challenge test. The notches of the Minimal Cross-Sectional area (MCA) are shown in acoustic measurements at pre- and post-challenge of the allergen (blue and red lines, respectively) in cases of vehicle treatment (a) and fexofenadine treatment (b). Because of nasal congestion, the post-challenge MCA decreased compared to the pre-challenge MCA in both cases. However, the extent of the MCA decrease was smaller in fexofenadine administration (b) than in vehicle administration (a). Rhinoscopic im-ages at post-challenge in cases of vehicle administration (c) and fexofenadine admin-istration (d). The tip of the distal end of the ethmoturbinate is visible in the case of fexofenadine administration (red circle, (d)).

because of congestion (Figure 1(c)). To quantify the extent of nasal congestion measured by an acoustic rhinometer, "MCA Change" was defined as (postchallenge $\mathrm{MCA} /$ pre-challenge $\mathrm{MCA}$ ) \%. In vehicle administration, "MCA Change" decreased to $<40 \%$, while fexofenadine administration significantly restored "MCA Change" to $>60 \%(\mathrm{n}=4, p<0.05$, Figure 2$)$. Thus, fexofenadine significantly ameliorated post-challenge nasal congestion, although the recovery was not complete.

Fexofenadine is an inverse agonist of the histamine $\mathrm{H} 1$ receptor, and it functions by blocking constitutive activation of receptors stimulated by histamine [11]. The $\mathrm{H} 1$ receptor consists of an active and inactive conformation coexisting in equilibrium. As an inverse agonist, the drug stabilizes the inactive conformation of the $\mathrm{H} 1$ receptor, causing a shift in the equilibrium toward the inactive 




Figure 2. Nasal congestion ameliorated by fexofenadine. Data were expressed as "MCA Change" (post-challenge MCA/pre-challenge MCA) \%. Data represent the mean \pm standard deviation $(S D)$. Open bar: vehicle administration $(n=4)$, solid bar: fexofenadine administration $(\mathrm{n}=4), p<0.05$.

state. Therefore, frequent and prophylactic use of fexofenadine effectively blocks active $\mathrm{H} 1$ receptor-mediated signaling. In the present study, even a single dose of fexofenadine demonstrated amelioration of nasal congestion. Higher doses and repeated administration of fexofenadine are expected to diminish nasal congestion to greater extent.

In conclusion, an acoustic rhinometry technique for the quantitative assessment of nasal congestion was successfully applied for the first time in an allergic model of cynomolgus macaques sensitized with Japanese cedar pollen. Using this technique and model, the ameliorative effect of an antihistamine drug, fexofenadine, was evaluated on nasal obstructionin the early phase response of allergic rhinitis.

\section{Acknowledgements}

We are grateful to Mr. Ryoji Kato (Yushin Precision Equipment Co., Ltd.) and Mr. Toshihito Akashi (Department of Medical Equipment, Finggal Link Co., Ltd.) for generous assistance on rhinometry. We also thank the staff at Intelligence and Technology Lab. Inc. for their excellent animal handling and supporting the experiment.

\section{References}

[1] Storms, W. (2008) Allergic Rhinitis-Induced Nasal Congestion: Its Impact on Sleep Quality. Primary Care Respiratory Journal, 17, 7-18. https://doi.org/10.3132/pcrj.2008.00001

[2] Okubo, K., Kurono, Y., Ichimura, K., Enomoto, T., Okamoto, Y., Kawauchi, H., et al. (2017) Japanese Guidelines for Allergic Rhinitis 2017. Allergology International, 66, 205-219. https://doi.org/10.1016/j.alit.2016.11.001

[3] Okubo, K., Okamasa, A., Honma, G. and Komatsubara, M. (2015) Safety and Efficacy of Fluticasone Furoate Nasal Spray in Japanese Children 2 to $<15$ Years of Age with Perennial Alergic Rhinitis: A Multicentre, Open-Label Trial. Allergoloty International, 64, 60-65. 
[4] Fisher, E.W., Lund, V.J. and Scadding, G.K. (1994) Acoustic Rhinometry in Rhinological Practice: Discussion Paper. Journal of the Royal Society of Medicine, 87, 411-413.

[5] Roithmann, R., Cole, P., Chapnik, J., Shpirer, I., Hoffstein, V. and Zamel, N. (1995) Acoustic Rhinometry in the Evaluation of Nasal Obstruction. Laryngoscope, 105, 275-281. https://doi.org/10.1288/00005537-199503000-00010

[6] Kaise, T., Akamatsu, Y., Ohmori, K., Ishii, A. and Karasawa, A. (2001) Effects of Olopatadine Hydrochloride on the Release of Thromboxane B2 and Histamine from Nasal Mucosa after Antigen-Antibody Reaction in Guinea Pigs. Allergology International, 50, 337-341. https://doi.org/10.1046/j.1440-1592.2001.00236.x

[7] Straszek, S.P., Taagehøj, F., Graff, S. and Pedersen, O.F. (2003) Acoustic Rhinometry in Dog and Cat Compared with a Fluid-Displacement Method and Magnetic Resonance Imaging. Journal of Applied Physiology, 95, 635-642.

https://doi.org/10.1152/japplphysiol.01105.2002

[8] Kawasaki, H., Kaise, T., Manabe, H. and Watanabe, A. (2005) The Phosphodiesterase 4 Inhibitor Prevents Antigen-Induced Biphasic Nasal Obstruction in Brown Norway Rats. Allergology International, 54, 427-433.

https://doi.org/10.2332/allergolint.54.427

[9] Shorey-Kendrick, L.E., Ford, M.M., Allen, D.C., Kuryatov, A., Lindstrom, J., Wilhelm, L., et al. (2015) Nicotinic Receptors in Non-Human Primates: Analysis of Genetic and Functional Conservation with Humans. Neuropharmacology, 96, 263-273. https://doi.org/10.1016/j.neuropharm.2015.01.023

[10] Chamanza, R., Taylor, I., Gregori, M., Hill, C., Swan, M. and Goodchild, J. (2016) Normal Anatomy, Histology, and Spontaneous Pathology of the Nasal Cavity of the Cynomolgus Monkey (Macaca fascicularis). Toxicologic Pathology, 44, 636-654. https://doi.org/10.1177/0192623315626523

[11] Jutel, M., Akdis, M. and Akdis, C.A. (2009) Histamine, Histamine Receptors and their Role in Immune Pathology. Clinical and Experimental Allergy, 39, 1786-1800. https://doi.org/10.1111/j.1365-2222.2009.03374.X 